\title{
THE ALIQUOT CONSTANT
}

\author{
WIEB BOSMA AND BEN KANE
}

\begin{abstract}
The average value of $\log s(n) / n$ taken over the first $N$ even integers is shown to converge to a constant $\lambda$ when $N$ tends to infinity; moreover, the value of this constant is approximated and proven to be less than 0 . Here $s(n)$ sums the divisors of $n$ less than $n$. Thus the geometric mean of $s(n) / n$, the growth factor of the function $s$, in the long run tends to be less than 1 . This could be interpreted as probabilistic evidence that aliquot sequences tend to remain bounded.
\end{abstract}

\section{InTRODUCTION}

This paper is concerned with the average growth of aliquot sequences. An aliquot sequence is a sequence $a_{0}, a_{1}, a_{2}, \ldots$ of positive integers obtained by iteration of the sum-of-aliquot-divisors function $s$, which is defined for $n>1$ by

$$
s(n)=\sum_{\substack{d \mid n \\ d<n}} d .
$$

The aliquot sequence with starting value $a_{0}$ is then equal to

$$
a_{0}, \quad a_{1}=s\left(a_{0}\right), \quad a_{2}=s\left(a_{1}\right)=s^{2}\left(a_{0}\right), \quad \ldots ;
$$

we will say that the sequence terminates (at 1) if $a_{k}=s^{k}\left(a_{0}\right)=1$ for some $k \geq 0$. The sequence cycles (or is said to end in a cycle) if $s^{k}\left(a_{0}\right)=s^{l}\left(a_{0}\right)$ for some $k, l$ with $0 \leq l<k$, where $s^{0}(n)=n$ by definition.

Note that $s$ is related to the ordinary sum-of-divisors function $\sigma$, with $\sigma(n)=\sum_{d \mid n} d$, by $s(n)=\sigma(n)-n$ for integers $n>1$.

The main open question in this area can be phrased as: does every aliquot cycle remain bounded? That is, does every aliquot sequence terminate (at 1) or cycle, or do sequences exist that grow unbounded? The conjecture that all sequences remain bounded is often referred to as the Catalan-Dickson conjecture.

The origin of this paper lies in computational work done to test integer factorization routines for the computer algebra system Magma[1].

Date: December 18, 2009. 
The most efficient known method to compute $s(n)$ uses the multiplicativity of $\sigma$ and requires the factorization of $n$. Iterating $s$ provides long sequences of more or less random numbers of similar size, and this property is useful in testing factorization methods. It was noticed that for even starting values the aliquot sequences tend to increase or decrease in size fairly slowly, by an amount that seemed constant over different starting values, whereas sequences with odd starting values usually terminate quickly.

Around 1996 Andrew Granville [6] furnished a proof for this phenomenon; see Theorem (3.3) and Theorem (3.7) below. Further computation seemed to suggest that the constant $\lambda$ involved would be smaller (but only just!) than 0, but no proof of this was obtained. Recently, we were able to obtain estimates that are good enough to prove this property.

Altogether this led to the main result of this paper.

Theorem 1.1. The geometric mean $\mu$ of $\frac{s(2 n)}{2 n}$ over all positive integers $n$ exists, and equals

$$
\mu=e^{-0.03 \cdots} .
$$

In particular, the aliquot growth factor $\mu=0.969 \cdots<1$.

Roughly summarizing, this means: on average, even aliquot sequences tend to decrease in size! In some sense this may be taken as probabilistic evidence in favour of the Catalan-Dickson conjecture.

This paper is built up as follows. After some preliminaries, we state and prove the convergence of the geometric mean for even values; also, an expression for the resulting constant $\lambda=\log \mu$ as a difference of $\alpha$ (closely related to the growth of $\sigma(n) / n$ ) and $\beta$ is derived. In the next section an easy upper bound for $\alpha$ (which involves a sum over all prime numbers) is given. The final section is devoted to a lower bound for $\beta$; this is trickier, as it involves an infinite sum of terms that themselves are infinite products over all primes.

\section{Elementary observations}

Although $\sigma(n)>n$ for $n>1$, all three possibilities $s(n)<n, s(n)=n$ and $s(n)>n$ for $s$ do occur: $s(p)=1$ for prime numbers, and in general $s\left(p^{k}\right)=1+p+\cdots+p^{k-1}<p^{k}$ for prime powers; $s(P)=P$ for perfect numbers $P=2^{p-1}\left(2^{p}-1\right)$ (with $2^{p}-1$ prime), and $s(n)>n$ for $n=P \cdot q$, where $P$ is perfect and $q$ any odd prime other than $2^{p}-1$, since $\sigma(P q)=2 P(q+1)$.

Besides terminating at 1 after hitting a prime, or ending in a perfect number, it is also possible that an aliquot sequence ends in a cycle of 
length 2 or more: amicable numbers are pairs $m, n$ for which $\sigma(m)=$ $m+n=\sigma(n)$, hence $s(m)=n$ and $s(n)=m$, and a 2-cycle is formed. Sociable numbers form cycles of larger length (and are known only for length 4, 5, 6, 8, 9 and 28; see [10]). For more on these cycles, including historical remarks, see also [8].

Note that

$$
\sigma(n)=\prod_{p^{k} \| n}\left(1+p+\cdots+p^{k}\right)
$$

is multiplicative, while $s(n)=\sigma(n)-n$ is not. A useful observation is that

$$
\frac{\sigma(2 n)}{2 n} \geq \frac{3}{2}
$$

by multiplicativity of $\sigma$ and since $\frac{\sigma\left(2^{k}\right)}{2^{k}} \geq \frac{3}{2}$ for $k \geq 1$.

Also note that $1+p+\cdots+p^{k}$ (for prime $p$ ) is only odd when $p$ is odd and $k$ is even. Hence $\sigma(n)-n$ for odd $n$ will only be even if $n$ is a square, and for even $n$ it will only be odd if $n$ is a square or twice a square. Hence: unless an accidental square (or twice a square) occurs, parity is preserved in aliquot sequences! In this sense $s$ does not behave randomly at all.

In fact, divisibility by (even) perfect numbers also tends to persist, and Guy and Selfridge [7] studied other types of persistence as well, but we will ignore all but the parity aspect and only consider even and odd aliquot sequences separately.

We are interested in the growth of the sequence $n, s(n), s^{2}(n), \ldots$, in other words, in the question of whether $s(n) / n$ tends to be smaller or greater than 1.

Apparently, Wunderlich (in [15]) was the first to state the following result explicitly; the first statement (formulated for $\sigma$ rather than $s$ ) appears already in [5].

\section{Theorem 2.2. Theorem}

$$
\begin{aligned}
\lim _{N \rightarrow \infty} \frac{1}{N} \sum_{n=1}^{N} \frac{s(n)}{n} & =\frac{\pi^{2}}{6}-1=0.6449 \ldots \\
\lim _{N \rightarrow \infty} \frac{1}{N} \sum_{n=1}^{N} \frac{s(2 n)}{2 n} & =\frac{5 \pi^{2}}{24}-1=1.0562 \ldots \\
\lim _{N \rightarrow \infty} \frac{1}{N} \sum_{n=1}^{N} \frac{s(2 n-1)}{2 n-1} & =\frac{3 \pi^{2}}{24}-1=0.2337 \ldots
\end{aligned}
$$


As

$$
\sum_{n=1}^{N} \frac{s(2 n-1)}{2 n-1}=\sum_{\substack{n=1 \\ n \text { odd }}}^{2 N}\left(\frac{\sigma(n)}{n}-1\right)
$$

we find by the same argument usually given for the computation of $\zeta(2)$, that

$$
\lim _{N \rightarrow \infty} \sum_{n=1}^{N} \frac{1}{N} \frac{s(2 n-1)}{2 n-1}=\sum_{\substack{x=1 \\ x \text { odd }}}^{2 N} \frac{1}{x^{2}}-1=\frac{3}{4} \zeta(2)-1 .
$$

from which the whole theorem follows.

Based on the second statement in Theorem (2.2), Guy and Selfridge seem to have drawn the conclusion that even aliquot sequences will tend to grow unbounded (see [7] page 103). Just like a sequence in which the terms are alternately multiplied by 5 and by $\frac{1}{5}$ will remain bounded although the average growth factor tends to 2.6, we cannot draw the conclusion that even aliquot sequences tend to grow unbounded from the fact that the average of $s(2 n) / 2 n$ exceeds 1 . What really matters is not the arithmetic mean, but rather the geometric mean:

$$
\sqrt[N]{\prod_{n=1}^{N} \frac{s(n)}{n}}=\exp \left(\frac{1}{N} \sum_{n=1}^{N} \log (s(n) / n)\right) .
$$

Remark 2.3. To draw conclusions about the Catalan-Dickson conjecture, one needs more than just the arithmetic or geometric mean of $\sigma(n) / n$. Davenport [3] showed that there exists a continuous function of $t$ giving the natural density of $t$-abundant numbers satisfying $\sigma(n) / n \geq t$. See [13], and [11, for recent progress on this function. This needs to be combined with the persistance of drivers as in [7].

A good approximation is known for the value of the function at $t=2$, implying that $s(n) / n$ exceeds 1 for a little less than a quarter of all $n$, see [4].

Finally, note that these questions relate to deep problems, as it is known [12] that the Riemann hypothesis is equivalent to the statement that $\sigma(n) / n$ is bounded by $e^{\gamma} \log \log n$ for all $n \geq 5041$; see also [2], 9], [14] on this connection.

\section{The Aliquot constant}

We first show the following result on the geometric mean for the ordinary sum of divisors function. 


\section{Proposition 3.1.}

$\frac{1}{N} \sum_{n \leq N} \log \frac{\sigma(n)}{n}=A+O\left(\frac{1}{\log x}\right)$, with $A=\sum_{p \text { prime }} \alpha(p) \approx 0.4457$,

where

$$
\alpha(p)=\left(1-\frac{1}{p}\right) \sum_{m \geq 1} \frac{1}{p^{m}} \log \left(1+\frac{1}{p}+\cdots+\frac{1}{p^{m}}\right) .
$$

Proof. Taking the product over all powers $p^{m}$ dividing $n$, with $p$ prime and $m \geq 1$, we have

$$
\frac{\sigma(n)}{n}=\prod_{p^{m} \mid n} \frac{\sigma\left(p^{m}\right) / p^{m}}{\sigma\left(p^{m-1}\right) / p^{m-1}},
$$

and hence

$$
\begin{aligned}
\sum_{n \leq x} \log \frac{\sigma(n)}{n} & =\sum_{n \leq x} \sum_{p^{m} \mid n} \log \frac{p^{m+1}-1}{p\left(p^{m}-1\right)}=\sum_{p^{m} \leq x} \log \frac{p^{m+1}-1}{p\left(p^{m}-1\right)}\left[\frac{x}{p^{m}}\right] \\
& =x \sum_{p^{m} \leq x} \frac{1}{p^{m}} \log \frac{p^{m+1}-1}{p\left(p^{m}-1\right)}+O\left(\frac{x}{\log x}\right) .
\end{aligned}
$$

For a fixed prime $p$ we have

$$
\frac{p^{m+1}-1}{p\left(p^{m}-1\right)}=1+\frac{p-1}{p\left(p^{m}-1\right)}=1+O\left(\frac{1}{p^{m}}\right),
$$

SO

$$
\begin{aligned}
& \sum_{p^{m} \leq x} \frac{1}{p^{m}} \log \frac{p^{m+1}-1}{p\left(p^{m}-1\right)}=\sum_{p^{m} \leq x} \frac{1}{p^{m}} \log \frac{1-1 / p^{m+1}}{1-1 / p^{m}} \\
& =-\frac{1}{p} \log \left(1-\frac{1}{p}\right)+\left(\frac{1}{p}-\frac{1}{p^{2}}\right) \log \left(1-\frac{1}{p^{2}}\right)+\cdots+O\left(\frac{1}{x^{2}}\right) \\
& =\left(1-\frac{1}{p}\right)\left(\frac{1}{p} \log \left(1+\frac{1}{p}\right)+\frac{1}{p^{2}} \log \left(1+\frac{1}{p}+\frac{1}{p^{2}}\right)+\cdots\right)+O\left(\frac{1}{x^{2}}\right)
\end{aligned}
$$

and the result follows.

Theorems 3.3 and 3.7 are our main asymptotic results on the growth of aliquot sequences. Roughly speaking, they state that the growth factor diverges to 0 when considered over all starting values, whereas confined to even values it converges, to $\lambda$.

Theorem 3.3.

$$
\frac{1}{N} \sum_{n=1}^{N} \log \frac{s(n)}{n}=-e^{-\gamma} \log \log N+O(\log \log \log N) .
$$


Proof. As $s(n) \geq 1$ for $n>1$ we have

$$
\frac{s(n)}{n} \geq \frac{1}{p_{1}}
$$

for the smallest prime factor $p_{1}$ of $n$. Thus

$$
\begin{aligned}
\sum_{1<n \leq x} \log \frac{s(n)}{n} \geq-\sum_{1 \leq n \leq x} \log p_{1}(n) \geq-\sum_{p \leq x} \log p \sum_{\substack{n \leq x \\
q \mid \frac{n}{p} \Rightarrow q \geq p}} 1 \\
\geq-\sum_{p \leq x} \log p \cdot \#\left\{m \leq \frac{x}{p}: m \text { has its prime factors } \geq p\right\} .
\end{aligned}
$$

By the small sieve: if $p=x^{\frac{1}{u}}$ then

$$
\begin{aligned}
\#\left\{m \leq \frac{x}{p}: q \mid m \Rightarrow q \geq p\right\} & =\prod_{q<p}\left(1-\frac{1}{q}\right) \frac{x}{p}\left(1+O\left(e^{-2 u}\right)+\frac{1}{\log x}\right) \\
& =\frac{e^{-\gamma} x}{p \log p}\left(1+O\left(\frac{1}{\log p}+e^{-2 u}\right)\right) .
\end{aligned}
$$

Hence

$$
\begin{aligned}
\sum_{1<n \leq x} \log \frac{s(n)}{n} & \geq-e^{-\gamma} x \sum_{p \leq x} \frac{1}{p}+O\left(x \sum_{p \leq x} \frac{1}{p \log p}+x \sum_{p \leq x} \frac{1}{p} e^{-u}\right) \\
& \geq-e^{-\gamma} x \log \log x+O(x) .
\end{aligned}
$$

On the other hand, let $M$ be the set of integers of the form $m p \leq x$ where all prime factors of $m$ are $\geq p \log x$. For such integers we have $\omega(m) \leq \log (x) / \log (p \log x)$,

$$
\frac{\sigma(m p) / m p}{\sigma(p) / p} \leq \prod_{q \mid m}\left(1-\frac{1}{q}\right)^{-1} \leq\left(1-\frac{1}{p \log x}\right)^{-\omega(m)} \leq 1+O\left(\frac{1}{p \log \log x}\right),
$$

so

$$
\frac{\sigma(m p)}{m p}-1 \leq \frac{1}{p}\left(1+O\left(\frac{1}{\log \log x}\right)\right)
$$

Then

$$
\begin{aligned}
\sum_{1<n \leq x} \log \frac{s(n)}{n} & =\sum_{1<n \leq x} \log \left(\frac{\sigma(n)}{n}-1\right) \\
& \leq \sum_{1<n \leq x} \log \frac{\sigma(n)}{n}-\sum_{n \in M}\left(\log p+O\left(\frac{1}{\log \log p}\right)\right) .
\end{aligned}
$$


Let $M^{\prime}$ be the set of integers $m p \in M$ with $p \leq x^{\frac{1}{U}}$ where $U$ is a large fixed number. With $y=e^{(\log \log x)^{2}}$ we find:

$$
\begin{aligned}
& \sum_{n \in M} \log p \geq \sum_{n \in M^{\prime}} \log p \geq \sum_{y \leq p \leq x \frac{1}{U}} \log p \cdot \#\left\{m \leq \frac{x}{p}: q \mid m \Rightarrow q>p \log x\right\} \\
& \geq \sum_{y \leq p \leq x} \log p \cdot \prod_{q \leq p \log x}\left(1-\frac{1}{q}\right) \frac{x}{p}\left(1+O\left(e^{-U}+\frac{1}{\log x}\right)\right) \\
& \geq \sum_{y \leq p \leq x} \frac{e^{-\gamma} x}{p}\left(1+O\left(e^{-U}+\frac{1}{\log x}\right)\right) \\
&(3.6) \quad \geq e^{-\gamma} x(\log \log x+O(\log \log \log x)) .
\end{aligned}
$$

Combining (3.6) and (3.5) with (3.4) and Proposition (3.1) we obtain

$$
\sum_{1 \leq n \leq x} \log \frac{s(n)}{n}=-e^{-\gamma} x \log \log x+O(x \log \log \log x) .
$$

\section{Theorem 3.7.}

$$
\frac{1}{N} \sum_{n=1}^{N} \log \frac{s(2 n)}{2 n}=\lambda+O(1 / \log N)
$$

where

$$
\lambda=\alpha(2)+\sum_{p \text { prime }} \alpha(p)-\sum_{j \geq 1} \frac{1}{j}\left(\left(2 \beta_{j}(2)-1\right) \prod_{\substack{p \geq 3 \\ \text { prime }}} \beta_{j}(p)\right),
$$

with

$$
\alpha(p)=\left(1-\frac{1}{p}\right) \sum_{m \geq 1} \frac{1}{p^{m}} \log \left(1+\frac{1}{p}+\cdots+\frac{1}{p^{m}}\right),
$$

as before, and

$$
\beta_{j}(p)=\left(1-\frac{1}{p}\right) \sum_{m \geq 0} \frac{1}{p^{m}}\left(1+\frac{1}{p}+\cdots+\frac{1}{p^{m}}\right)^{-j}
$$

Proof. Suppose that $J$ is sufficiently large. Then

$$
\begin{array}{r}
\sum_{n \leq x} \log \left(\frac{\sigma(2 n)}{2 n}-1\right)=\sum_{n \leq x} \log \frac{\sigma(2 n)}{2 n}+\sum_{n \leq x} \log \left(1-\frac{2 n}{\sigma(2 n)}\right) \\
=\sum_{n \leq x} \log \frac{\sigma(2 n)}{2 n}-\sum_{j=1}^{J} \frac{1}{j} \sum_{n \leq x}\left(\frac{2 n}{\sigma(2 n)}\right)^{j}+O\left(\frac{x}{(3 / 2)^{J}}\right),
\end{array}
$$


using (2.1). Proceeding as before we get

$$
\sum_{n \leq x} \log \frac{\sigma(2 n)}{2 n}=A^{*} x+O\left(\frac{x}{\log x}\right)
$$

where

$$
A^{*}=\sum_{p} \alpha(p)+\sum_{m \geq 1} \frac{1}{2^{m+1}}\left(\log 2+\log \left(1-\frac{1}{2^{m+1}}\right)\right) .
$$

We now use the following result.

Lemma 3.12. Let $f$ be a multiplicative function with $0 \leq f\left(p^{m}\right) \leq 1$ with $1-f\left(p^{m}\right) \ll \frac{1}{p}$ for every prime $p$. Then

$$
\sum_{n \leq x} f(n)=x \prod_{p}\left[\left(1-\frac{1}{p}\right) \sum_{m \geq 0} \frac{f\left(p^{m}\right)}{p^{m}}\right]+O\left((\log x)^{C}\right) .
$$

Proof. Let $g\left(p^{m}\right)=f\left(p^{m}\right)-1 \ll \frac{1}{p}$, and $g(n)=\prod_{p^{m} \|_{n}} g\left(p^{m}\right)$. Then

$$
\begin{aligned}
\sum_{n \leq x} f(n) & =\sum_{n \leq x} \prod_{p^{m} \| n}\left(1+g\left(p^{m}\right)\right)= \\
& =\sum_{n \leq x} \sum_{\substack{d \mid n \\
\operatorname{gcd}(d, n / d)=1}} g(d)=\sum_{d \leq x} g(d) \sum_{\substack{m \leq x / d \\
\operatorname{gcd}(m, d)=1}} 1= \\
& =\sum_{d \leq x} g(d) \frac{\phi(d)}{d} \frac{x}{d}+O\left(\sum_{d \leq x} g(d) 2^{\nu(d)}\right)= \\
& =x \prod_{p}\left(1+\sum_{m \geq 1} \frac{g\left(p^{m}\right)}{p^{m}} \frac{\phi\left(p^{m}\right)}{p^{m}}\right)+O\left((\log x)^{C}\right) .
\end{aligned}
$$

But

$$
\begin{aligned}
1+\sum_{m \geq 1} \frac{g\left(p^{m}\right)}{p^{m}} \frac{\phi\left(p^{m}\right)}{p^{m}} & =1+\left(1-\frac{1}{p}\right) \sum_{m \geq 1} \frac{f\left(p^{m}\right)-1}{p^{m}}= \\
& =\left(1-\frac{1}{p}\right) \sum_{m \geq 0} \frac{f\left(p^{m}\right)}{p^{m}}
\end{aligned}
$$

and the Lemma follows.

We apply this to $f_{j}(n)=\left(\frac{3 n}{\sigma(2 n)}\right)^{j}$; then

$$
f_{j}\left(p^{m}\right)=\left(\frac{p^{m}}{\sigma\left(p^{m}\right)}\right)^{j}
$$


for $p$ an odd prime, and

$$
f_{j}\left(2^{m}\right)=\left(\frac{3 \cdot 2^{m}}{\sigma\left(2^{m+1}\right)}\right)^{j}=\left(\frac{3}{2}\right)^{j}\left(\frac{2^{m+1}}{\sigma\left(2^{m+1}\right)}\right)^{j} .
$$

But then by the Lemma

$$
\begin{aligned}
& \frac{1}{j} \sum_{n \leq x}\left(\frac{2 n}{\sigma(2 n)}\right)^{j}=\frac{1}{j} \frac{1}{(3 / 2)^{j}} \sum_{n \leq x} f_{j}(n)= \\
= & \frac{x}{j} \frac{1}{(3 / 2)^{j}} \prod_{p}\left\{\left(1-\frac{1}{p}\right)\left(\sum_{m \geq 0} \frac{f_{j}\left(p^{m}\right)}{p^{m}}\right)\right\}+O\left((\log x)^{c}\right)= \\
= & \frac{x}{j}\left(2 \beta_{j}(2)-1\right)\left(\prod_{\substack{p \geq 3 \\
\text { prime }}} \beta_{j}(p)\right)+O\left((\log x)^{c}\right) .
\end{aligned}
$$

Collecting the information we get

$$
\sum_{n \leq x} \log \left(\frac{\sigma(2 n)}{2 n}-1\right)=\lambda x+O\left(\frac{1}{\log x}\right),
$$

and the result follows.

Example 3.14. Although most of the rest of this paper is devoted to a numerical estimate for $\lambda$, necessary because of the behaviour of $\beta$, it is easy to see the convergence of $\lambda$. If we sum $s(n) / n$ for the first even values of $n$, up to $N$, we get the following.

\begin{tabular}{|c|c|}
\hline$N$ & $\sum_{2 n \leq N} s(2 n) /(2 n)$ \\
\hline $10^{2}$ & $-0.0567457527 \ldots$ \\
$10^{3}$ & $-0.0356519058 \ldots$ \\
$10^{4}$ & $-0.0335201796 \ldots$ \\
$10^{5}$ & $-0.0332873082 \ldots$ \\
$10^{6}$ & $-0.0332626444 \ldots$ \\
$10^{7}$ & $-0.0332598642 \ldots$ \\
$10^{8}$ & $-0.0332595156 \ldots$ \\
$3.953 \cdot 10^{9}$ & $-0.0332597045 \ldots$ \\
\hline
\end{tabular}

\section{Computing $\alpha$}

By definition,

$$
\lambda=2 \alpha(2)+\sum_{\substack{p \geq 3 \\ \text { prime }}} \alpha(p)-\sum_{j \geq 1} \frac{1}{j}\left((2 \beta(2)-1) \cdot \prod_{\substack{p \geq 3 \\ \text { prime }}} \beta_{j}(p)\right) .
$$


In this section we will compute a good approximation and upper bound for:

$$
\alpha=2 \alpha(2)+\sum_{\substack{p \geq 3 \\ p \text { prime }}} \alpha(p) .
$$

First note that for any prime $p$

$$
\left(1-\frac{1}{p}\right) \sum_{m \geq 1} \frac{1}{p^{m}} \log \left(1+\frac{1}{p}+\cdots \frac{1}{p^{m}}\right)=\sum_{m \geq 1} \frac{1}{p^{m}} \log \left(\frac{1+p+\cdots p^{m}}{p+\cdots+p^{m}}\right) .
$$

Since for all $m \geq 1$

$$
\frac{1+p+\cdots+p^{m}}{p+\cdots+p^{m}}=1+\frac{1}{p+\cdots+p^{m}} \leq 1+\frac{1}{p^{m}}
$$

and $\log (1+x) \leq x$ for all $x>0$, we find that the tail

$$
\sum_{m=M+1}^{\infty} \frac{1}{p^{m}} \log \left(\frac{1+p+\cdots+p^{m}}{p+\cdots+p^{m}}\right)
$$

is bounded by

$$
\frac{1}{p^{M+1}}\left(1+\frac{1}{p}+\frac{1}{p^{2}}+\cdots\right) \cdot \log \left(1+\frac{1}{p^{M+1}}\right) \leq \frac{p}{p-1}\left(\frac{1}{p^{M+1}}\right)^{2} .
$$

We will denote this bound by

$$
A(p, M)=\frac{p}{p-1}\left(\frac{1}{p^{M+1}}\right)^{2} .
$$

It also follows that, for any $N \geq 1$

$$
\sum_{\substack{p>N \\ p \text { prime }}} \alpha(p) \leq \sum_{\substack{p>N \\ p \text { prime }}} \sum_{m \geq 1} \frac{1}{p^{m}} \log \left(1+\frac{1}{p^{m}}\right) \leq \sum_{n>N} \frac{1}{n} \log \left(1+\frac{1}{n}\right) .
$$

But

$$
\sum_{n>N} \frac{1}{n} \log \left(1+\frac{1}{n}\right) \leq \int_{N}^{\infty} \frac{1}{x} \log \left(1+\frac{1}{x}\right) d x=\int_{0}^{\frac{1}{N}} \frac{1}{z} \log (1+z) d z
$$

is clearly bounded by $\frac{1}{N}$.

Theorem 4.1. For any $N>2$ and $L, M>1$ :

$$
\begin{aligned}
\alpha & \leq \sum_{m=1}^{L} \frac{1}{2^{m}} \log \left(1+\frac{1}{2}+\cdots+\frac{1}{2^{m}}\right)+2 A(2, L)+ \\
& +\sum_{\substack{p \leq N \\
p \text { oddprime }}} \sum_{m=1}^{M} \frac{1}{p^{m}} \log \left(\frac{1+\cdots+p^{m}}{p+\cdots+p^{m}}\right)+\sum_{\substack{3 \leq p \leq N \\
p \text { prime }}} A(p, M)+\frac{1}{N} .
\end{aligned}
$$


As the sums in this theorem are all finite this gives us an effective way to compute an upper bound on $\alpha$.

Example 4.2. In the table below we have listed the outcome of some computations for $\alpha$ with $L=M=15$. These computations were done within half an hour (including the primality tests for all primes up to $10^{8}$ ) on an ordinary PC, using Magma.

\begin{tabular}{|c|c|c|}
\hline$N$ & sums & error bound \\
\hline $10^{4}$ & $0.6983072233 \ldots$ & $1.0000093132 \ldots \cdot 10^{-4}$ \\
$10^{5}$ & $0.6983162365 \ldots$ & $1.0000931323 \ldots \cdot 10^{-5}$ \\
$10^{6}$ & $0.6983169710 \ldots$ & $1.0009313233 \ldots \cdot 10^{-6}$ \\
$10^{7}$ & $0.6983170329 \ldots$ & $1.0093132338 \ldots \cdot 10^{-7}$ \\
$10^{8}$ & $0.6983170383 \ldots$ & $1.0931323384 \ldots \cdot 10^{-8}$ \\
\hline
\end{tabular}

Corollary 4.3. $\alpha<0.69831705$.

\section{Computing $\beta$}

Our next goal is to compute a lower bound for

$$
\beta=\sum_{j \geq 1} \frac{1}{j}\left(\left(2 \beta_{j}(2)-1\right) \prod_{\substack{p>2 \\ \text { prime }}} \beta_{j}(p)\right)
$$

where for every prime $p$

$$
\beta_{j}(p)=\left(1-\frac{1}{p}\right)\left(1+\sum_{m \geq 1} \frac{1}{p^{m}}\left(\frac{1}{1+\frac{1}{p}+\frac{1}{p^{2}}+\cdots+\frac{1}{p^{m}}}\right)^{j}\right)
$$

Observe that for $j \geq 1$

$$
\begin{aligned}
\beta_{j}(p) & =\sum_{m \geq 0} \frac{1}{p^{m}}\left(\frac{p^{m}}{\sigma\left(p^{m}\right)}\right)^{j}-\sum_{m \geq 0} \frac{1}{p^{m+1}}\left(\frac{p^{m}}{\sigma\left(p^{m}\right)}\right)^{j} \\
& =1+\sum_{m \geq 1} \frac{1}{p^{m}} \frac{\left(p^{m}+\cdots+p^{2 m-1}\right)^{j}-\left(p^{m-1}+\cdots+p^{2 m-1}\right)^{j}}{\sigma\left(p^{m}\right)^{j} \sigma\left(p^{m-1}\right)^{j}} \\
& =1-\sum_{m \geq 1}\left(\frac{1}{p^{m}} \frac{\sum_{k=1}^{j}\left(\begin{array}{l}
j \\
k
\end{array}\right)\left(p^{m-1}\right)^{k}\left(p^{m} \sigma\left(p^{m-1}\right)\right)^{j-k}}{\sigma\left(p^{m}\right)^{j} \sigma\left(p^{m-1}\right)^{j}}\right)
\end{aligned}
$$




$$
\begin{aligned}
& =1-\sum_{m \geq 1}\left(\frac{1}{p^{m}} \sum_{k=1}^{j} \frac{\left(\begin{array}{l}
j \\
k
\end{array}\right) p^{m j-k} \sigma\left(p^{m-1}\right)^{j-k}}{\sigma\left(p^{m}\right)^{j} \sigma\left(p^{m-1}\right)^{j}}\right) \\
& =1-\sum_{m \geq 1}\left(\frac{1}{p^{m}}\left(\frac{p^{m}}{\sigma\left(p^{m}\right)}\right)^{j} \sum_{k=1}^{j} \frac{\left(\begin{array}{l}
j \\
k
\end{array}\right)}{\left(p \sigma\left(p^{m-1}\right)\right)^{k}}\right) \\
& =\sum_{m \geq 0}(-1)^{\nu\left(p^{m}\right)} g_{j}\left(p^{m}\right) h_{j}\left(p^{m}\right),
\end{aligned}
$$

where the multiplicative function $\nu(n)$ denotes the number of different prime divisors of $n$, and we set for non-negative $m$

$$
g_{j}\left(p^{m}\right)=\frac{1}{p^{m}}\left(\frac{p^{m}}{\sigma\left(p^{m}\right)}\right)^{j},
$$

(in particular $g_{j}(1)=1$ ), and for positive $m$

$$
h_{j}\left(p^{m}\right)=\sum_{k=1}^{j} \frac{\left(\begin{array}{l}
j \\
k
\end{array}\right)}{\left(p \sigma\left(p^{m-1}\right)\right)^{k}},
$$

while $h_{j}(1)=1$ by definition.

If we extend our definition to

$$
g_{j}(n)=\frac{1}{n}\left(\frac{n}{\sigma(n)}\right)^{j}
$$

for any positive integer $n$, we find

$$
g_{j}(n)=\prod_{p^{m} \| n} g_{j}\left(p^{m}\right)
$$

where $n=\prod_{p^{m} \| n} p^{m}$ is the factorization of $n$. We also define $h_{j}$ for all positive integers $n$ by multiplicativity

$$
h_{j}(n)=\prod_{p^{m} \| n} h_{j}\left(p^{m}\right) .
$$

If we define, for composite $n$,

$$
\beta_{j}(n)=(-1)^{\nu(n)} g_{j}(n) h_{j}(n)
$$

then for every positive integer $n$, we find

$$
\prod_{\substack{p \geq 2 \\ p \text { prime }}} \beta_{j}(p)=\sum_{n \in \mathbb{N}} \beta_{j}(n) .
$$

This way, the infinite product in the definition of $\beta$, (5.1), is replaced by an infinite sum; note, however, the slight complication caused by 
the factor $2 \beta_{j}(2)-1$ rather than $\beta_{j}(2)$ in this definition. Since

$$
2 \beta(2)-1=\sum_{m \geq 1} \frac{1}{2^{m}}\left(\frac{1}{1+\frac{1}{2}+\frac{1}{2^{2}}+\cdots+\frac{1}{2^{m}}}\right)^{j}=\sum_{m \geq 1} g_{j}\left(2^{m}\right)
$$

we obtain

$$
\beta=\sum_{j \geq 1} \frac{1}{j}\left(\sum_{\substack{n \in \mathbb{N} \\ n \text { even }}} \beta_{j}^{*}(n)\right)
$$

where

$$
\beta_{j}^{*}(n)=g_{j}\left(2^{k}\right) \beta_{j}\left(n_{o}\right) \quad \text { for } n=2^{k} n_{o} \text {, with } n_{o} \text { odd. }
$$

To get a lower bound for $\beta$ we will replace these sums by finite summations for bounded $j$ and $n$, and bound the remaining terms. As clearly $\beta_{j}(p)>0$ for odd $p$, and so is $2 \beta_{j}(2)-1$ by (5.3), we see from the definition that for any $J \geq 1$

$$
\beta \geq \sum_{j=1}^{J} \frac{1}{j}\left(\sum_{\substack{n \in \mathbb{N} \\ n \text { even }}} \beta_{j}^{*}(n)\right)
$$

Define for real $e>0$

$$
S_{j, e}=\left\{n \in \mathbb{N}: h_{j}(n)>\frac{1}{n^{e}}\right\} .
$$

Since

$$
\begin{aligned}
\sum_{\substack{n=2^{k_{n}} \\
n_{o}>N, n_{o} \notin S_{j, e} \\
n_{0}}} \beta_{j}^{*}(n) & =\sum_{\substack{n=2^{k} n_{n_{o} \text { even }} \\
n_{o}>N, n_{o} \notin S_{j, e}}} \frac{1}{n}\left(\frac{n}{\sigma(n)}\right)^{j} h_{j}\left(n_{o}\right) \leq \\
& \leq \sum_{\substack{n_{o}>N \\
\text { odd }}}\left(\sum_{k=1}^{\infty} \frac{1}{2^{k} n_{o}}\left(\frac{2^{k} n_{o}}{\sigma\left(2^{k} n_{o}\right)}\right)^{j}\right) h_{j}\left(n_{o}\right) \leq \\
& \leq\left(\frac{2}{3}\right)^{j} \frac{1}{2} \int_{x=N}^{\infty} \frac{1}{x^{1+e}} d x=\frac{1}{2 e} \frac{1}{N^{e}}\left(\frac{2}{3}\right)^{j},
\end{aligned}
$$

we immediately obtain the following result.

Lemma 5.6. For any $J \geq 1$ and even $N_{j}>1($ for $j=1,2 \ldots, J)$ :

$$
\beta \geq\left(\sum_{j=1}^{J} \frac{1}{j} \sum_{\substack{n \leq N_{j} \text { or } n \in S_{j, e} \\ n \text { even }}} \beta_{j}^{*}(n)\right)-\sum_{j=1}^{J} \frac{1}{2 j e N_{j}^{e}}\left(\frac{2}{3}\right)^{j} .
$$


Define for $e, c>0$

$$
T_{j}^{e, c}=\left\{(p, m): h_{j}\left(p^{m}\right) \geq \frac{1}{c \cdot\left(p^{m}\right)^{e}}, p \text { prime, } m \in \mathbb{Z} \text { with } m \geq 1\right\}
$$

Now $T_{j}^{e, c}$ is finite whenever $0<e<1$ (let $p^{m} \rightarrow \infty$ in the definition). Put

$$
M_{j, e}=\max _{n \in \mathbb{Z} \geq 1} h_{j}(n) n^{e}
$$

note that $M_{j, e} \geq 1$

Lemma 5.7. Let $0<e<1$. If $n \in S_{j, e}$ then for $m \geq 1$

$$
p^{m} \| n \quad \Rightarrow \quad(p, m) \in T_{j}^{e, M_{j, e}}
$$

in particular, $S_{j, e}$ is finite.

Proof. Let $M=M_{j, e}$. Suppose that $n \in S_{j, e}$, and write $n=n_{1} \cdot n_{2}$ with $\operatorname{gcd}\left(n_{1}, n_{2}\right)=1$, such that if $p^{m} \| n_{1}$ then $(p, m)$ is in $T_{j}^{e, M}$ and if $p^{m} \| n_{2}$ then $(p, m) \notin T_{j}^{e, M}$.

If $n_{2}>1$ then

$$
h_{j}(n) n^{e}=h_{j}\left(n_{1}\right) n_{1}^{e} \cdot h_{j}\left(n_{2}\right) n_{2}^{e} \leq M \cdot \frac{1}{M}=1,
$$

contradicting our assumption $n \in S_{j, e}$. Thus $n_{2}=1$ and the lemma is proved.

This means that the inner sums in Lemma (5.6) are finite.

Algorithm 5.8. This results in the following method for computing a lower bound for $\beta$.

(1) Choose $J \geq 1$, and perform the following three steps for $j=$ $1,2, \ldots, J$.

(2) Choose an even integer $N_{j}>1$ large enough.

(3) Compute $\frac{1}{j} \sum_{\substack{n \leq N_{j} \\ \text { even }}} \beta_{j}^{*}(n)$, for example from the definitions (5.5) and (5.2).

(4) Determine the set $S_{j, e}$ as follows:

(4a) Choose $e=e_{j}$ and determine $T_{j, e, 1}$. Then compute $M_{j, e}=$ $\max _{n} h_{j}(n) n^{e}$ by choosing the product of the worst value for $(p, m) \in T_{j, e, 1}$ for each prime $p$ occurring in this set.

(4b) Choose $c=c_{j}$ and determine $T_{j, e, c}$.

(4c) Determine $S_{j, e}$, that is, the positive integers $n$ built up from prime powers $p^{m}$ with $(p, m)$ in $T_{j, e, c}$, for which $h_{j}(n)>$ $1 / n^{e}$

and compute $\frac{1}{j} \sum_{\substack{n \in S_{j, e} \\ n>\text { Neven }}} \beta_{j}^{*}(n)$. 
(5) Compute the error term $\left(2 j e N_{j}^{e}\right)^{-1} \cdot\left(\frac{2}{3}\right)^{j}$.

(6) Take the sum of results from Steps 3 and 4, and subtract the sum of the results of Step 5, taken over $j=1,2, \ldots, J$.

\begin{tabular}{|l|l|r|r|r|r|}
\hline$j$ & $e$ & $\# S$ & main & contribution $S$ & error \\
\hline 1 & 1 & 0 & 0.508058 & & $4.18 \cdot 10^{-12}$ \\
2 & 0.75 & 71678431 & 0.134230 & $4.7096 \cdot 10^{-12}$ & $2.99 \cdot 10^{-9}$ \\
3 & 0.60 & 139189128 & 0.048944 & $8.949 \cdot 10^{-12}$ & $3.276 \cdot 10^{-7}$ \\
4 & 0.48 & 93183633 & 0.020684 & $9.7488 \cdot 10^{-12}$ & $2.462 \cdot 10^{-6}$ \\
5 & 0.35 & 10201152 & 0.009564 & $-9.1679 \cdot 10^{-12}$ & $2.66 \cdot 10^{-5}$ \\
6 & 0.28 & 27662520 & 0.004706 & $-1.95 \cdot 10^{-12}$ & $7.89 \cdot 10^{-5}$ \\
7 & 0.20 & 24415897 & 0.002425 & $-3.315 \cdot 10^{-12}$ & $3.31 \cdot 10^{-4}$ \\
8 & 0.15 & 65291514 & 0.001295 & $5.907 \cdot 10^{-12}$ & $7.26 \cdot 10^{-4}$ \\
9 & 0.03 & 7466778 & 0.000711 & $-9.511 \cdot 10^{-12}$ & $2.59 \cdot 10^{-2}$ \\
\hline$\sum_{j=1}^{8}$ & & & 0.729906 & $<10^{-10}$ & $1.1625 \cdot 10^{-4}$ \\
\hline
\end{tabular}

Example 5.9. Take $N=10^{9}$. The table lists for $j=1,2, \ldots, 9$ the values of $e, \# S, M$, the main term from Step (3) in the algorithm, the contribution from $S$ in Step (4) and the error term from Step (5).

As an indication of the size of the numbers involved: the largest element of $S_{2,0.75}$ is the 24-digit product of the 18 primes less than 62 . Since the error term for $j=9$ exceeds the contribution of the main term, we have not included this one in the final sum.

As a consequence, we find that $\beta \geq 0.728743$.

\section{Corollary 5.10.}

$$
\lambda<-0.030 \text {. }
$$

\section{REFERENCES}

[1] Wieb Bosma, John Cannon, and Catherine Playoust, The Magma algebra system. I. The user language, J. Symbolic Comput., 24 (1997), 235-265.

[2] Keith Briggs, Abundant numbers and the Riemann hypothesis, Experiment. Math. 15 (2006), 251-256.

[3] Harold Davenport, Über numeri abundantes, Preuss. Akad. Wiss. Sitzungsberichte (1933), 830-837.

[4] M. Deléglise, Bounds for the density of abundant integers, Experiment. Math. 7 (1998), 137-143.

[5] G. Lejeune Dirichlet, Über die Bestimmung der mittleren Werthe in der Zahlentheorie, Abhandlungen der Königlich Preussischen Akademie von 1849, 69-83 (= Werke, zweiter Band, 49-66).

[6] Andrew Granville, personal communication.

[7] Richard K. Guy, J.L. Selfridge, What drives an aliquot sequence?, Math. Comp. 29 (1975), 101-107; Corrigendum: Math. Comp. 34 (1980), 319-321. 
[8] Mitsuo Kobayashi, Paul Pollack, Carl Pomerance, On the distribution of sociable numbers, J. Number Theory 129 (2009), 1990-2009.

[9] J.C. Lagarias, An elementary problem equivalent to the Riemann hypothesis, Amer. Math. Monthly 109 (2002), 534-543.

[10] http://djm.cc/sociable.txt

[11] Y.-F.S. Pétermann, An $\Omega$-theorem for an error term related to the sum-ofdivisors functions, Monatsh. Math. 103 (1987), 145-157.

[12] G. Robin, Grandes valeurs de la fonction somme des diviseurs et hypothèse de Riemann, J. Math. Pures Appl. 63 (1984), 187-213.

[13] Andreas Weingartner, The distribution functions of $\sigma(n) / n$ and $n / \phi(n)$, Proc. Amer. Math. Soc. 135 (2007), 2677-2681.

[14] Marek Wjtowicz, Robin's inequality and the Riemann hypothesis, Proc. Japan Acad. 83 Ser. A (2007), 47-49.

[15] M. C. Wunderlich, The aliquot project: an application of job chaining in number theoretic computing, Proceedings of the third ACM symposium on Symbolic and algebraic computation (1976), 276-284.

Department of Mathematics, Radboud Universiteit, Nijmegen NL

E-mail address: $\{$ W.Bosma, B.Kane\}@math.ru.nl 